\title{
Ultrasound-guided spinal stereotactic system for intraspinal implants
}

\author{
Amirali Toossi, MSc, ${ }^{1,7}$ Dirk G. Everaert, PhD, ${ }^{2,7}$ Peter Seres, MSc, ${ }^{3}$ Jacob L. Jaremko, MD, PhD, ${ }^{4}$ \\ Kevin Robinson, PT, DSc, ${ }^{5}$ C. Chris Kao, MD, PhD, ${ }^{6}$ Peter E. Konrad, MD, PhD, ${ }^{6,7}$ and \\ Vivian K. Mushahwar, $\mathrm{PhD}^{1,2,7}$
}

\begin{abstract}
${ }^{1}$ Neuroscience and Mental Health Institute; ${ }^{2}$ Division of Physical Medicine and Rehabilitation, Department of Medicine; ${ }^{3}$ Department of Biomedical Engineering; ${ }^{4}$ Department of Radiology and Diagnostic Imaging, University of Alberta, Edmonton, Alberta, Canada; ${ }^{5}$ School of Physical Therapy, Belmont University; ${ }^{6}$ Department of Neurosurgery, Vanderbilt University Medical Center, Nashville, Tennessee; and ${ }^{7}$ Sensory Motor Adaptive Rehabilitation Technology (SMART) Network, University of Alberta, Edmonton, Alberta, Canada
\end{abstract}

OBJECTIVE The overall goal of this study was to develop an image-guided spinal stereotactic setup for intraoperative intraspinal microstimulation (ISMS). System requirements were as follows: 1) ability to place implants in various segments of the spinal cord, targeting the gray matter with a $<0.5-\mathrm{mm}$ error; 2) modularity; and 3) compatibility with standard surgical tools.

METHODS A spine-mounted stereotactic system was developed, optimized, and tested in pigs. The system consists of a platform supporting a micromanipulator with 6 degrees of freedom. It is modular and flexible in design and can be applied to various regions of the spine. An intraoperative ultrasound imaging technique was also developed and assessed for guidance of electrode alignment prior to and after electrode insertion into the spinal cord. Performance of the ultrasound-guided stereotactic system was assessed both in pigs ( 1 live and 6 fresh cadaveric pigs) and on the bench using four gelatin-based surrogate spinal cords. Pig experiments were conducted to evaluate the performance of ultrasound imaging in aligning the electrode trajectory using three techniques and under two conditions. Benchtop experiments were performed to assess the performance of ultrasound-guided targeting more directly. These experiments were used to quantify the accuracy of electrode alignment as well as assess the accuracy of the implantation depth and the error in spatial targeting within the gray matter of the spinal cord. As proof of concept, an intraoperative ISMS experiment was also conducted in an additional live pig using the stereotactic system, and the resulting movements and electromyographic responses were recorded.

RESULTS The stereotactic system was quick to set up ( $<10$ minutes) and provided sufficient stability and range of motion to reach the ISMS targets reliably in the pigs. Transverse ultrasound images with the probe angled at $25^{\circ}-45^{\circ}$ provided acceptable contrast between the gray and white matter of the spinal cord. In pigs, the largest electrode alignment error using ultrasound guidance, relative to the minor axis of the spinal cord, was $\leq 3.57^{\circ}$ (upper bound of the $95 \%$ confidence interval). The targeting error with ultrasound guidance in bench testing for targets $4 \mathrm{~mm}$ deep into the surrogate spinal cords was $0.2 \pm 0.02 \mathrm{~mm}$ (mean \pm standard deviation).

CONCLUSIONS The authors developed and evaluated an ultrasound-guided spinal stereotactic system for precise insertion of intraspinal implants. The system is compatible with existing spinal instrumentation. Intraoperative ultrasound imaging of the spinal cord aids in alignment of the implants before insertion and provides feedback during and after implantation. The ability of ultrasound imaging to distinguish between spinal cord gray and white matter also improves confidence in the localization of targets within the gray matter. This system would be suitable for accurate guidance of intraspinal electrodes and drug or cell injections.

https://thejns.org/doi/abs/10.3171/2018.1.SPINE17903

KEYWORDS intraspinal microstimulation; ultrasound imaging; patient-mounted stereotactic setup; spinal cord; swine model; targeting accuracy; surgical technique 
I NTRASPINAL microstimulation (ISMS) is a form of functional electrical stimulation ${ }^{29}$ in which arrays of fine microelectrodes are implanted within the spinal cord, targeting the ventral horns. Low-amplitude electrical pulses $(<100 \mu \mathrm{A})$ are delivered through the electrodes, activating neuronal networks and motor neuronal pools, resulting in behaviorally measured outcomes. ${ }^{2,23,24}$ To date, applications of ISMS have been mainly focused on restoring functions lost after spinal cord injury. Early clinical studies in humans utilized ISMS implants in the sacral segments of the spinal cord (S1-2) and showed successful bladder voiding in more than $75 \%$ of female patients (total of 13 patients) with bladder paralysis. ${ }^{28}$ Since then, preclinical studies have investigated the use of ISMS for the restoration of standing and walking, ${ }^{11,14,23,34}$ hand movements, ${ }^{37,40}$ and respiration ${ }^{21}$ in animal models. Intraspinal stimulation for standing and walking is one of the more widely studied types of ISMS in which the electrodes are implanted in the lumbosacral region of the spinal cord. Previous studies have shown promising functional outcomes for such implants and have motivated translation toward clinical testing. 2,11,23,34,38

An important requirement for clinical testing of ISMS is the development of a safe and precise implantation system. Precise manipulation and placement of ISMS microelectrodes into the spinal cord requires a stereotactic setup and an accurate targeting method. In restoring walking with ISMS, the cross-sectional area of the smallest target in the ventral horn of the feline spinal cord is approximately $0.2-0.3 \mathrm{~mm}^{2}$, corresponding to the tibialis anterior motor neuron pool..$^{25-27}$ The size of such a target for humans is not directly known; however, assuming that the functional activation size of the motor pool is proportional to the overall size of the spinal cord, homologous targets in the human spinal cord are likely approximately $30 \%$ larger. ${ }^{26,27,35}$

Existing stereotactic systems for intraspinal procedures can be categorized into two groups based on how they are mounted:20 1) on the surgical table ${ }^{16,33}$ or 2) on the spine of the patient. ${ }^{31}$ Systems mounted on the surgical table for positioning implants in the spinal cord have the risk of causing spinal cord trauma because of relative displacements of the surgical table and the spinal cord. Systems mounted on the spine of the patient alleviate this drawback.

Two spine-mounted stereotactic systems have been described in the literature. The first, called the "spinal derrick,"7,32 was developed for intraspinal stem cell delivery and has been tested clinically. This system involves fixation of a micromanipulator platform onto the patient's spine using four percutaneous laminar screws. ${ }^{32}$ In most of the preclinical and clinical studies using this system, estimation of the implant trajectory within the spinal cord was based on dorsal anatomical landmarks, micromanipulator coordinates, and MRI studies acquired prior to surgery. ${ }^{31,32}$ The targeting accuracy of this implant delivery system was not systematically reported; however, the reported injection targets in human spinal cords are in the ventral horns of the gray matter. These targets are at depths ranging from 3 to $5 \mathrm{~mm}$ from the dorsal surface ${ }^{30}$ and, unlike ISMS, may not require submillimeter target- ing accuracies. More recently, a prototype of an MRIcompatible spinal derrick was used for MRI-guided insertion of injection needles through the interlaminar space. ${ }^{13}$

The second spine-mounted stereotactic system was developed at the Mayo Clinic $^{9}$ for ISMS in pigs. The system anchors to the spine through eight MRI-compatible pedicle screws spanning four vertebral levels. Magnetic resonance imaging studies are obtained following the laminectomy and pedicle screw placement by using a custom MRI coil and MRI markers. The coil and markers are then removed, and a micromanipulator setup is mounted on the pedicle screws. The acquired MR images guide the coordinates on the micromanipulator system for targeting within the spinal cord. The reported targeting accuracy of this system in a bench setup is $1.09 \pm 0.2 \mathrm{~mm}$ (mean \pm standard deviation).

The required targeting accuracy for trajectory alignment of ISMS implants can be estimated based on the size of the spread of electrical current around the electrode tip. The functional stimulation amplitudes required for ISMS in cats range from 60 to $100 \mu \mathrm{A} . .^{11}$ For these stimulation amplitudes, the estimated radius of current spread is 0.4 and $0.5 \mathrm{~mm}$, respectively. ${ }^{1,25}$ Therefore, to ensure overlap between the intended electrode target and the electrically activated area, the spatial targeting error should be $<0.5$ $\mathrm{mm}$. A higher targeting accuracy minimizes the number of required electrode passes for successful stimulation and is critical for the safety and functional success of the implant. To date, the targeting accuracy goal of $0.5 \mathrm{~mm}$ has not been met by existing spine-mounted systems. ${ }^{9,32}$ This is due to the inherent visual illusions when eyeballing the trajectory of the electrode as well as the limitations in the spatial resolution of MR images obtained from real-time MRI-guided systems. Additionally, methods that only use MR images collected prior to the insertion of electrodes lack the ability to provide guidance and feedback during or after insertion.

We propose a novel spinal stereotactic system for ISMS that is mounted on the spine and for the first time relies on intraoperative ultrasound imaging for guiding the electrode trajectory into the spinal cord. Ultrasound is a desirable imaging modality for this task since it provides dynamic real-time imaging and is capable of providing very high spatial resolution. As a first application, we used the system for intraoperative ISMS implants. The specific aims of this study were to 1) develop a modular spinal stereotactic system with a minimal number of components that allows precise $3 \mathrm{D}$ targeting within the spinal cord gray matter, 2) develop an intraoperative ultrasound imaging method that allows visualization of the gray and white matter of the spinal cord as well as measurement of the electrode insertion angle, and 3) quantify electrode alignment accuracy using the spinal stereotactic system and ultrasound guidance in pigs and a benchtop setup using a surrogate spinal cord.

\section{Methods}

A spinal stereotactic system using ultrasound image guidance was developed and optimized through experiments on the bench and in pigs. 


\section{Spinal Stereotactic System}

This system provides a stereotactic framework that allows precise measurements in three dimensions and consists of three main components: a set of two metal frames (hereafter referred to as "loops"), a platform, and a micromanipulator (Fig. 1). The loops form an elevated base for attachment of the platform onto the spine, and the platform provides an adjustable base for the micromanipulator. Intraoperative ultrasound imaging is also used as part of this system to improve target selection and provide feedback during implantation procedures.

\section{Loops}

The loops are made of surgical spine rods (in this case, 5.5-mm diameter, Medtronic) that were shaped, under high heat, into a trapezoid-like shape and spot welded on one side. The loops can be closed (Fig. 1) or open (Fig. 2) and are mounted on the vertebrae with four pedicle screws. The trapezoid-like loops can have a range of dimensions: for ISMS implants, the bottom edge is shorter $(11 \mathrm{~cm})$ than the top $(17 \mathrm{~cm})$, and the height is $7 \mathrm{~cm}$. This construction reduces exposure of the bone in the craniocaudal direction while providing a sufficiently large working window at the top. The left and right loops can be stabilized laterally with length-adjustable cross-links that connect the loops across the top (in this case, $5.5-\mathrm{cm}$ maximal range). The open space between the L-shaped handle and the top bars of the open loops (Fig. 2) provides a wide working area in the transverse direction for ultrasound probe maneuvers.

\section{Platform}

The platform (in this case, $8.5 \mathrm{~cm}$ long $\times 8 \mathrm{~cm}$ wide) serves as a mounting base for the micromanipulator system, which attaches simply with an adaptor plate with a cross-shaped slit that slides over two screws in the platform (Fig. 2B and C). Once the micromanipulator is in the desired position, the two screws are tightened. The platform is designed to provide flexibility in the placement of the micromanipulator system in the lateral and craniocaudal directions. Flexibility in these directions is achieved by placing 6 rows of 10 screw holes in the platform. The cross-shaped slit in the adaptor plate further increases the lateral and craniocaudal placement options and allows the manipulator to be rotated in the coronal plane (Fig. 2B).

The platform is mounted on the loops with 2 "sliders." The 5-cm-long sliders have a longitudinal slit along the underside that fits over the loops (Fig. 2C). Two transverse screws with cone tips are tightened to clamp the sliders onto the loops.

\section{Micromanipulator System}

The micromanipulator system in this paper contains four commercially available components that we combined in order to obtain a sufficient range of motion in three directions of translation and two planes of rotation. The top stage is a Narishige MMN-33 micromanipulator (Narishige Company) with a $35-\mathrm{mm}$ range craniocaudally, $20 \mathrm{~mm}$ mediolaterally, and $37 \mathrm{~mm}$ dorsoventrally. The MMN-33 allows rotation in the transverse plane (Fig. 2D)

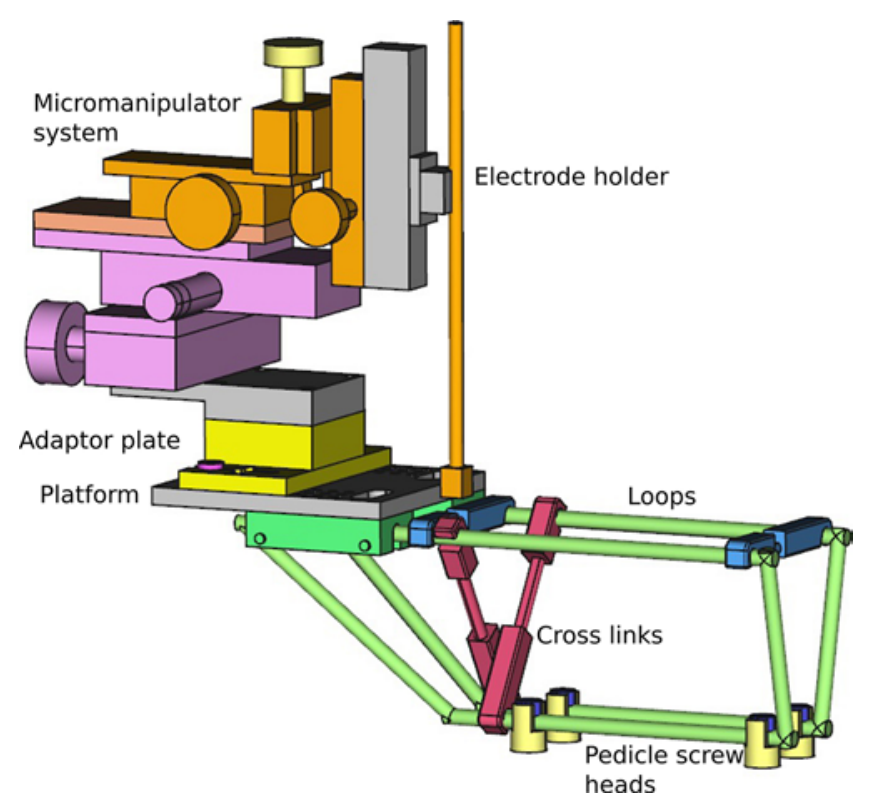

FIG. 1. The spinal stereotactic setup and its elements with special application to ISMS. Figure is available in color online only.

and is mounted on a 2D translation middle stage, which adds $60 \mathrm{~mm}$ in both the craniocaudal and mediolateral directions (Edmund Optics Inc.). The bottom stage is a goniometer (B54-40U2NR, Suruga Seiki Company), which provides $\pm 20^{\circ}$ of rotation in the sagittal plane (Fig. 2E).

\section{Mounting Procedure}

Pedicle screws are placed bilaterally in the pedicles of two neighboring vertebrae or two vertebrae skipping one level. The loops are placed in the swivel heads and loosely secured with the caps. A transverse cross-link is loosely attached to connect the two top bars, providing some lateral stability. The platform is loosely positioned on the loops, and the loops are adjusted so that the midline of the platform aligns with the spinal cord and the platform is parallel with the frontal plane of the body. The pedicle screw caps, cross-link, and platform screws are then securely tightened, locking all angles of the platform framework. The micromanipulator is then placed on the platform by sliding the adaptor plate under the top screws of the platform (Fig. 2B). The micromanipulator is rotated on the platform so that the main translation axis is aligned with the spinal cord (in the craniocaudal direction). The top screws of the platform are fastened, locking the micromanipulator in place.

\section{Intraoperative Ultrasound Imaging}

The ultrasound probe used in this study was an intraoperative Philips L15-7io linear array probe with a footprint of $23 \mathrm{~mm}$. The operating frequency of the probe was set to $15 \mathrm{MHz}$, and pixel size was $0.067 \times 0.067 \mathrm{~mm}$. The size of the probe allowed it to fit in the space above the spinal cord and scan it in the transverse plane during electrode insertion. The probe was not in contact with the cord and thus had to be submerged in saline, filling the spinal canal above the cord. 


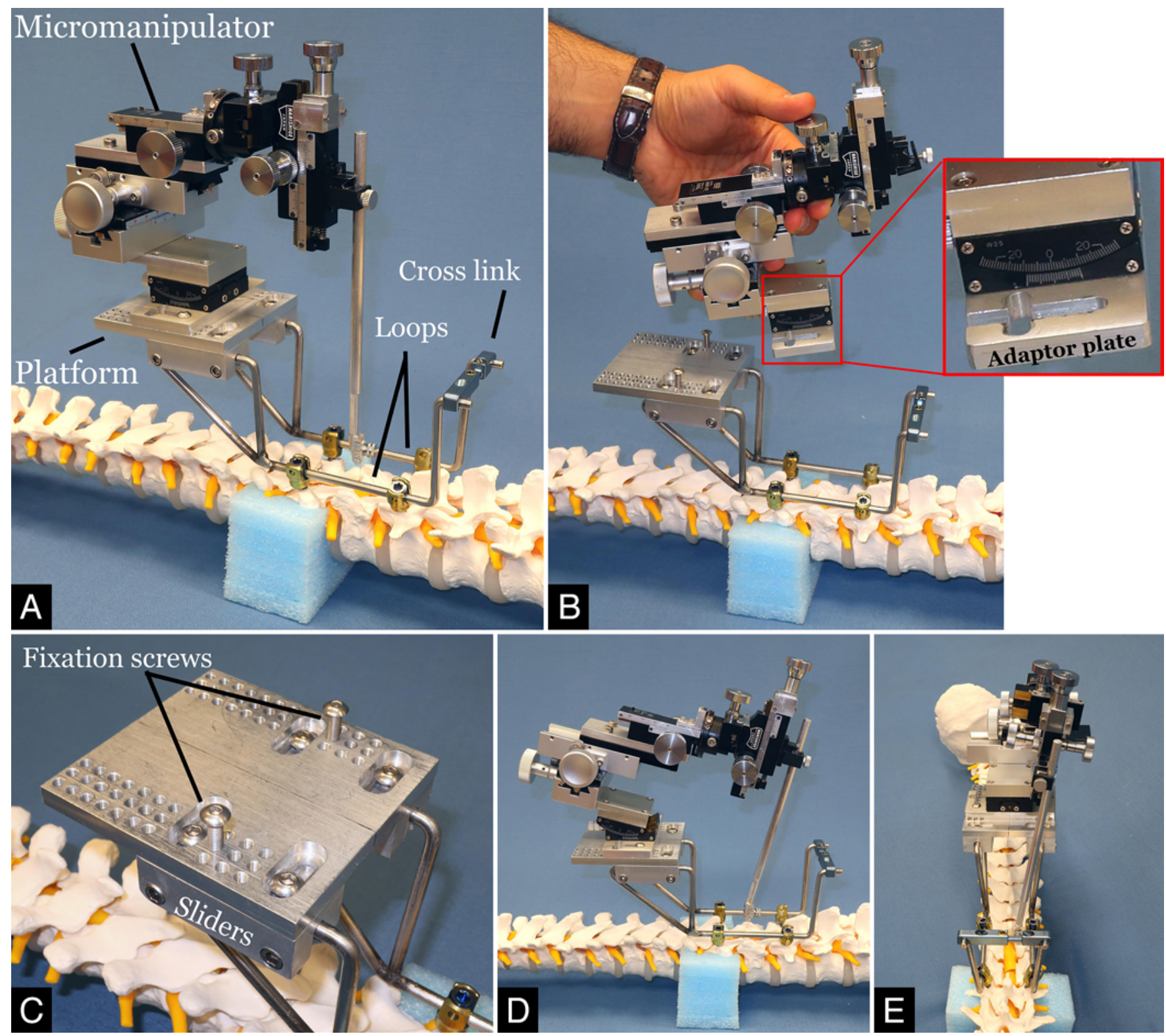

FIG. 2. A: Elements of the spinal stereotactic system. B: Micromanipulator placement onto the platform using an adaptor plate. The attachment allows for both translation in the craniocaudal direction and adjustment of angles in the coronal plane. C: The platform and its associated elements: fixation screws and sliders. D: Rotation of the electrode in the transverse plane using the stereotactic system. E: Rotation of the electrode holder and electrode in the sagittal plane using the stereotactic system. Figure is available in color online only.

\section{Surgery and Device Placement}

Animal experiments were conducted in 7 domestic pigs ( 6 freshly euthanized pigs and 1 live pig) with an average weight of $43.7 \pm 9.1 \mathrm{~kg}$. Pigs were the main animal model for this study because of the anatomical similarity of their spinal column and spinal cord to those of humans. ${ }^{4,36,38} \mathrm{All}$ animal experiments were conducted in accordance with protocols approved by the Institutional Animal Care and Welfare Committee at the University of Alberta.

The fresh pig cadavers were obtained after the animals were euthanized using an intravenous Euthanyl injection. In the experiment using the live animal, the pig was deeply anesthetized and maintained in the surgical plane throughout the duration of the experiment. In all animals, a laminectomy was performed to expose the lumbosacral enlargement of the spinal cord (lumbar vertebrae L4 to L6). Four multiaxial Medtronic pedicle screws (4.5-mm diameter $\times 25$-mm length) were placed bilaterally in the pedicles. The spinal stereotactic system was mounted onto the pedicle screws as described above. The dura mater and the arachnoid layers were then opened.

\section{Assessment of the Accuracy of Ultrasound-Guided Electrode Implantation in the Pig}

Intraoperative ultrasound imaging was used to guide the trajectory of glass-tube markers into the spinal cord prior 

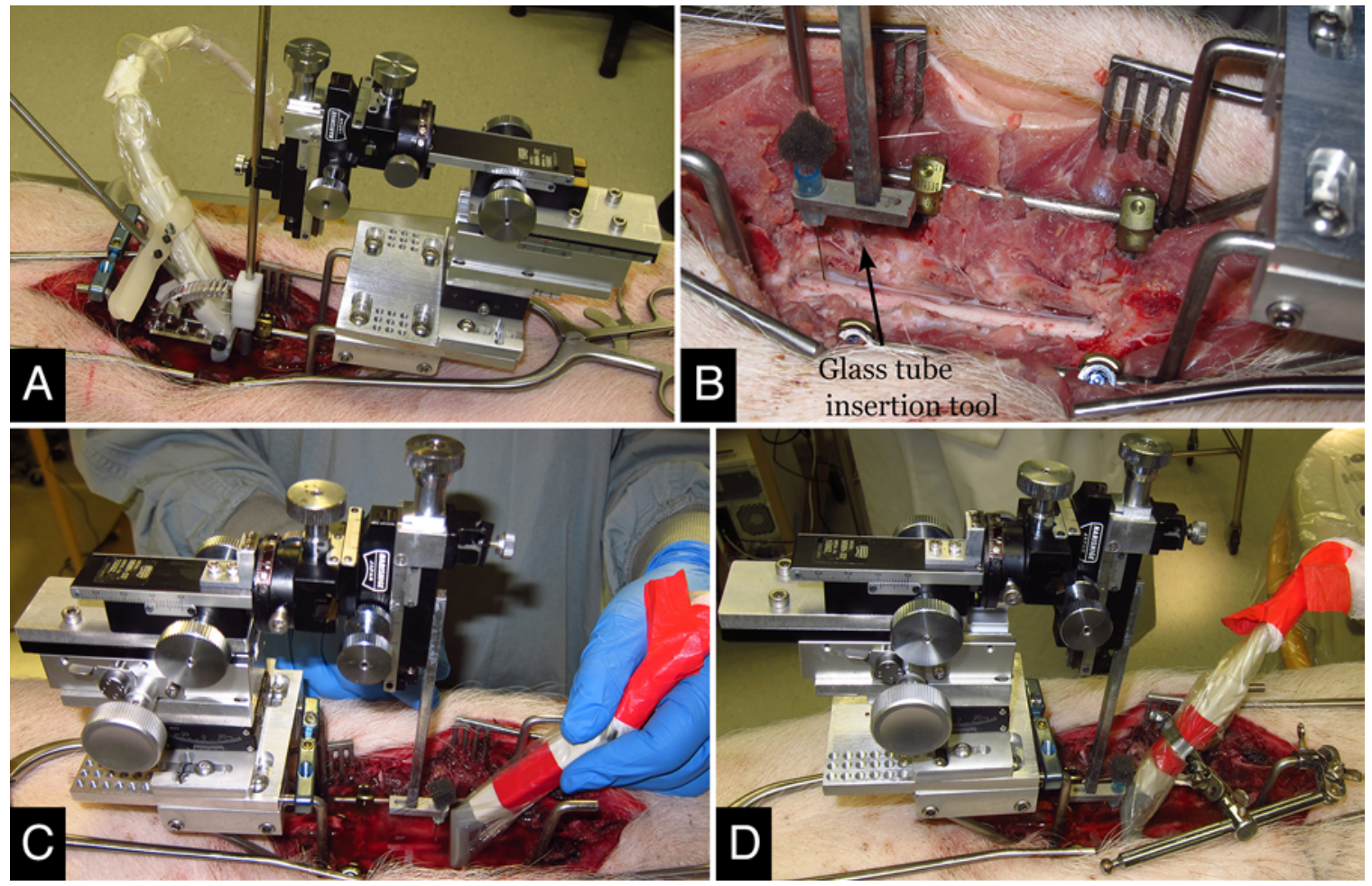

FIG. 3. The spinal stereotactic system used in a domestic pig. A: Customized setup for accurate modification of the ultrasound probe tilt angle in the sagittal plane. B: Glass-tube insertion tool. C: Ultrasound-guided electrode alignment with a handheld probe. D: Ultrasound-guided electrode alignment with the probe mounted onto the stereotactic setup. Figure is available in color online only.

to insertion. Markers were then implanted into the spinal cord using a custom-built insertion tool attached to the stereotactic system. After each experiment, the spinal cords were extracted and imaged using MRI. The MR images were used as the reference for measurement of the insertion angles of the implanted markers with respect to the major axis of the spinal cord. The targeting error of the ultrasound-guided implantation via the stereotactic system was assessed by comparing the insertion angles measured using ultrasound imaging and those measured using MRI.

\section{Ultrasound Visualization of Electrode and Spinal Cord}

In order to find the suitable range in the tilt angle of the ultrasound probe for viewing the gray and white matter of the spinal cord, a custom-made apparatus was used. As shown in Fig. 3A, orientation of the probe was varied in the transverse oblique scanning plane to obtain the best gray and white matter contrast in cross-sectional images of the spinal cord.

The suitable range in the probe tilt angle for visualizing the gray and white matter was $25^{\circ}-45^{\circ}$ from the short-axis view of the spinal cord. Tilt angles less than $25^{\circ}$ did not provide sufficient contrast between gray and white matter to distinguish between them. While tilting the probe in the transverse oblique plane improves gray-white discrimination, it distorts the shape of the spinal cord in the dorsoventral direction. Therefore, the smallest tilt angle providing the appropriate contrast is preferred. ${ }^{12}$ When visualizing the electrode and the spinal cord in one image, the ultrasound probe was positioned caudal to the electrode in the transverse oblique plane.

\section{Marker Implantation System}

Instead of implanting metal microelectrodes, which produce large artifacts in the MR images, small glass tubes were implanted in the spinal cord as markers that were visible in both the ultrasound and MR images. The glass-tube markers were approximately $5 \mathrm{~mm}$ long and had an outer diameter of $170 \mu \mathrm{m}$ (Wale Apparatus Company). The markers were inserted using a custom-built insertion tool that replaces the electrode stylus in the micromanipulator, as shown in Fig. 3B. The tool contains a holder for a 25 -gauge needle. Prior to each insertion, a glass tube was back-loaded into a needle, which was then placed in the holder. The needle was lowered by the micromanipulator and inserted $2 \mathrm{~mm}$ deep into the spinal cord. The glass tube was then inserted through the needle in the spinal cord using a tungsten wire acting as a plunger. The needle was then carefully retracted with the micromanipulator, leaving the tube implanted in the cord.

\section{Electrode Alignment in the Transverse Plane}

Traditionally for ISMS, surface landmarks such as the dorsal root entry zone are used as reference points to determine the laterality of the entry point of the electrode. Since ultrasound imaging can visualize the gray and white matter, it can also be used to guide and provide feedback on the laterality of the entry point of the electrodes. Suc- 


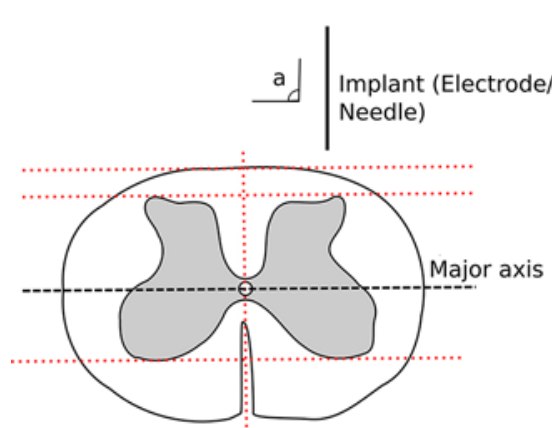

Transverse Plane

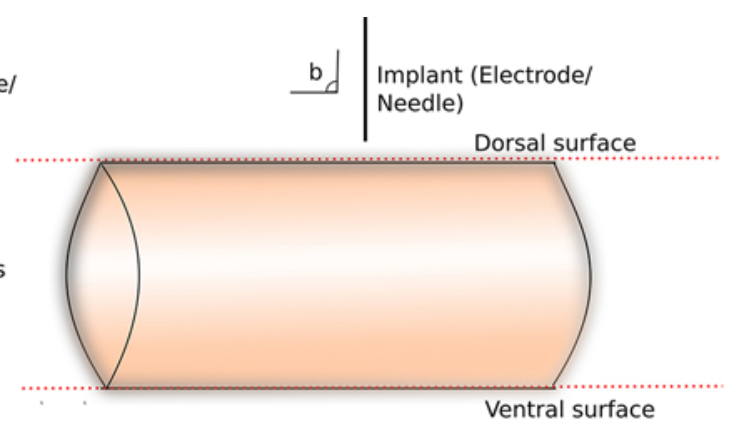

Sagittal Plane

FIG. 4. Spinal cord in the transverse (left) and sagittal (right) planes and the landmarks (dotted lines) that can be used to determine its orientation. In this study, the target for the electrode insertion angle ( $a$ and $b$ ) is $90^{\circ}$. Figure is available in color online only.

cessful projections from the entry point to the target in the ventral horn require the electrode to be inserted straight into the spinal cord. For this purpose, "straight" is defined as perpendicular to the major axis of the cord's elliptical shape. In the case of a perfectly symmetrical cord, this would mean the electrode was also perpendicular to the line connecting the tips of the ventral or dorsal horns and parallel with the anterior fissure (Fig. 4). Therefore, the goal in the experiments was to align the marker insertion needle perpendicularly to the cord's major axis, prior to insertion of the markers.

The angle of the insertion needle in the micromanipulator system was aligned using three methods: 1) eyeballing, 2) ultrasound guidance using a handheld probe (Fig. 3C), and 3) ultrasound guidance with the probe attached to the stereotactic setup (Fig. 3D). The probe was attached to the loops using a multi-jointed arm. These methods were investigated under two conditions: 1) no time limitation for the insertion of each marker and, for the eyeballing method, no limitation on the points of view used by the surgeon (referred to as the "unlimited-time condition"); and 2) time limit of 90 seconds for each in- sertion and, for the eyeballing method, the surgeon was asked to remain on their side of the patient, thus limiting the viewpoints to a more realistic condition (referred to as the "time-constrained condition"). In each experiment, at least 9 markers were implanted using each of the three alignment methods. Conditions 1 and 2 were each tested in three pigs.

The ultrasound-guided alignment of the insertion needle involved three steps: 1) tilting the probe to visualize the electrode and the spinal cord in one image; 2) identifying the orientation of the spinal cord (Fig. 5A and B) by drawing a line over the anterior fissure, drawing a line across the boundaries of the ventral or dorsal horns, and/ or drawing a tangent line over the dorsal surface of the spinal cord at the midline; and 3) adjusting the orientation of the electrode by rotating the micromanipulator in the transverse plane until the alignment becomes acceptable. The micromanipulator rotation stage was then locked, and an ultrasound image of the needle in its final position prior to implantation was taken to document the insertion angle (Fig. 5A and B). The marker was then implanted in the cord as described above. Tilting the ultrasound probe in
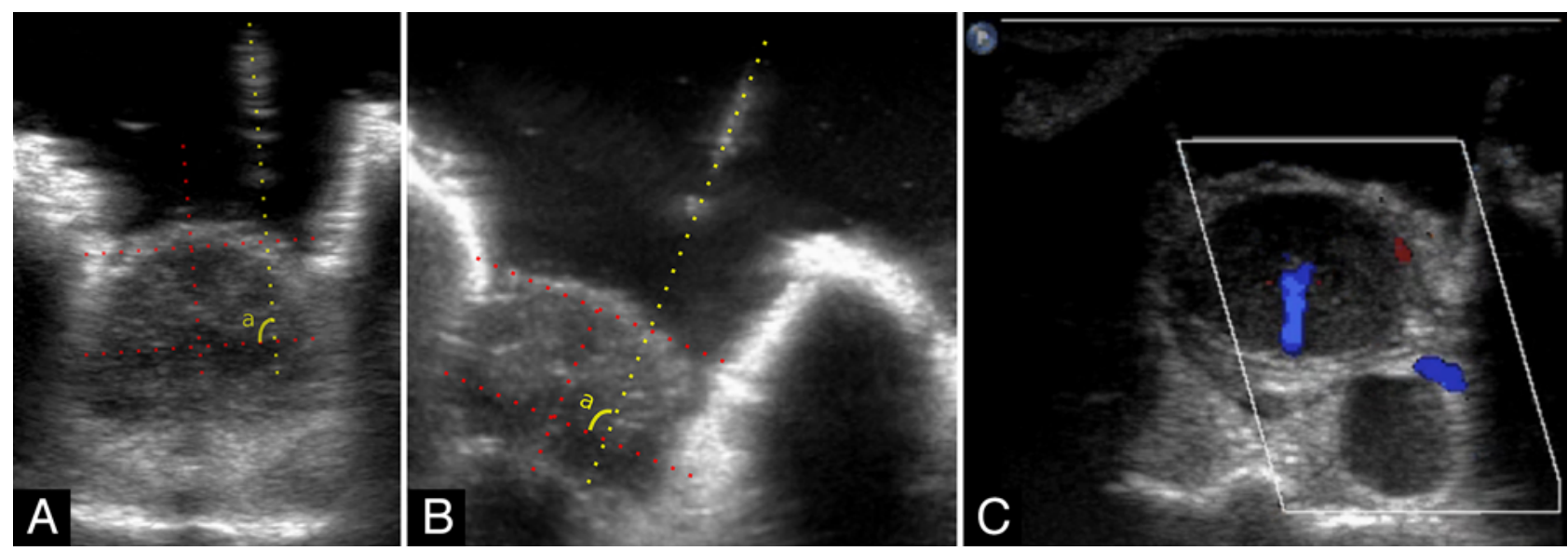

FIG. 5. Ultrasound images obtained from the lumbar enlargement of the pig spinal cord. A and B: Ultrasound-guided electrode alignment using the needle artifact and the spinal cord landmarks. C: Doppler ultrasound image of the spinal cord showing cerebrospinal fluid pulsations in the anterior fissure of the spinal cord. The orientation of the anterior fissure can be used to determine the orientation of the spinal cord. Figure is available in color online only. 


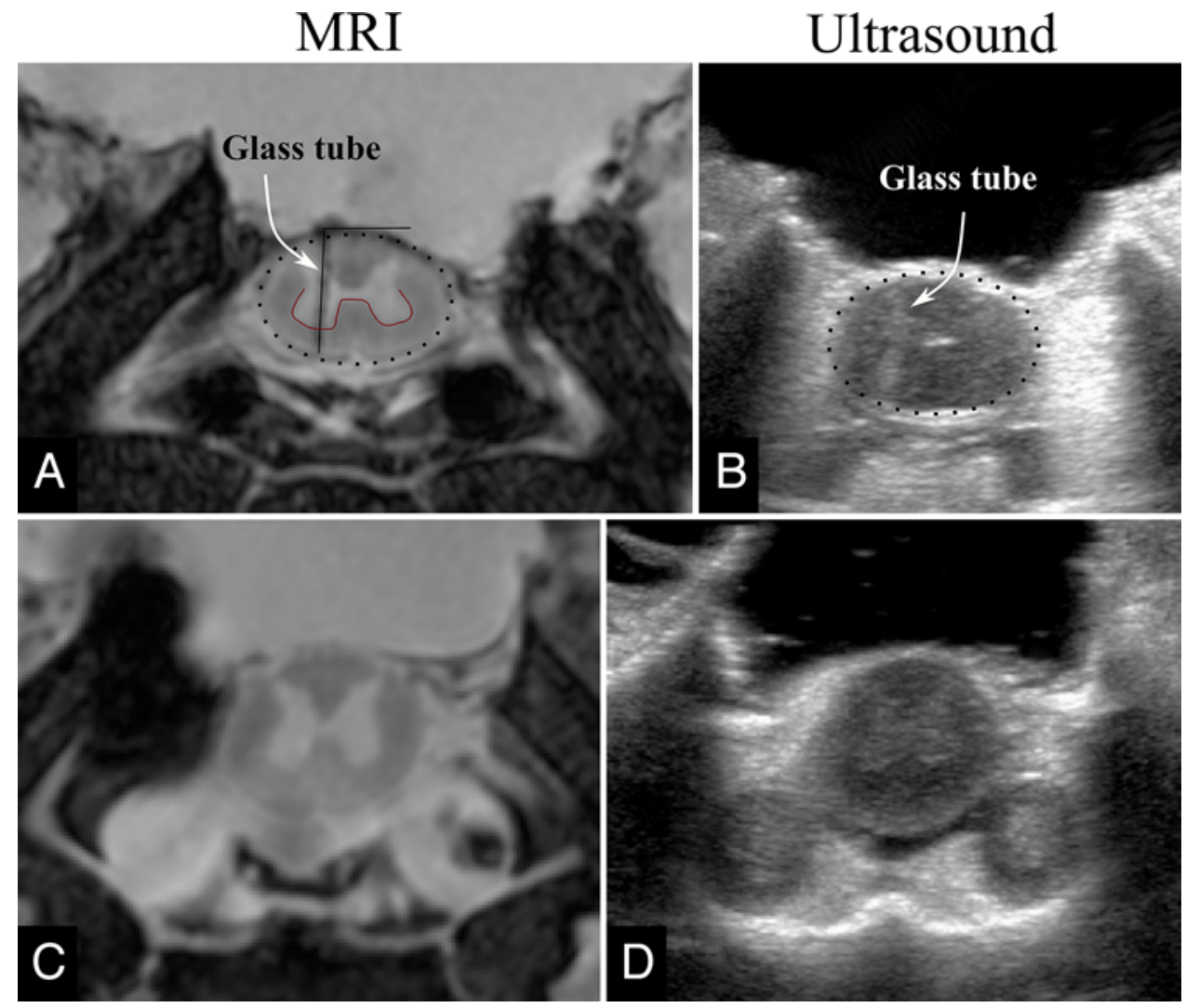

FIG. 6. Magnetic resonance and ultrasound images of similar segments of the spinal cord. Images in each row belong to the same slice of the spinal cord. A: Measurement of the insertion angle on an MR image. B: Ultrasound image showing an implanted glasstube marker. C: MR image of the spinal cord in the transverse plane showing the gray and white matter. D: Ultrasound image in the transverse plane of the spinal cord. Figure is available in color online only.

the transverse oblique plane did not affect the viewed orientation of the cross-section of the spinal cord. ${ }^{12}$

\section{Electrode Alignment in the Sagittal Plane}

The space was not sufficient for ultrasound imaging of the insertion in the sagittal plane because of the design of the glass-marker insertion tool (Fig. 3D). Therefore, an assisted eyeballing alignment method was used in the sagittal plane for all of the tested conditions. This method involved attaching a stylus to the micromanipulator and lowering it to the dorsal surface of the spinal cord. The stylus was then translated in the craniocaudal direction while adjusting the sagittal rotation angle until its translation path became parallel with the surface of the spinal cord.

In two experiments, after the markers were implanted into the spinal cord, the marker insertion tool was removed, and an ultrasound image of the marker in the sagittal plane of the spinal cord was recorded and the insertion angle was documented.

\section{Verification of Marker Placement Using MRI}

After all of the markers were inserted into the spinal cord, the animal (in the live pig experiment) was euthanized, and the lumbar spine (including the spinal cord) was extracted and fixed in formalin solution. The extracted spine was then scanned while submerged in saline using a 3-T Siemens Prisma MRI scanner at the Peter S. Allen MR Research Centre, University of Alberta. The MR images were acquired using a $1 \mathrm{Tx} / 15 \mathrm{Rx}$ knee coil and a 3D MEDIC sequence with a resolution of $0.25 \times 0.25 \times 1 \mathrm{~mm}$ in the transverse plane and $0.31 \times 0.31 \times 1 \mathrm{~mm}$ in the sagittal plane. The implanted markers in the spinal cord were identified in the MR images, and angle measurements were obtained using ImageJ software (National Institutes of Health; Fig. 6A).

\section{Statistical Analysis}

A two-way ANOVA was performed to analyze the effect of testing conditions (time constrained and unlimited time) and alignment methods (eyeballing, ultrasound guidance using a handheld probe, and ultrasound guidance using a mounted probe) on the alignment error of the electrode trajectory. A subsequent one-way ANOVA and Tukey Honestly Significant Difference post hoc analysis were performed for each testing condition to compare the three alignment techniques. We used t-tests to compare the two testing conditions for each alignment method. Differences were considered significant for $p \leq 0.05$. All analyses were performed using IBM SPSS software (version 22, IBM Corp.). 

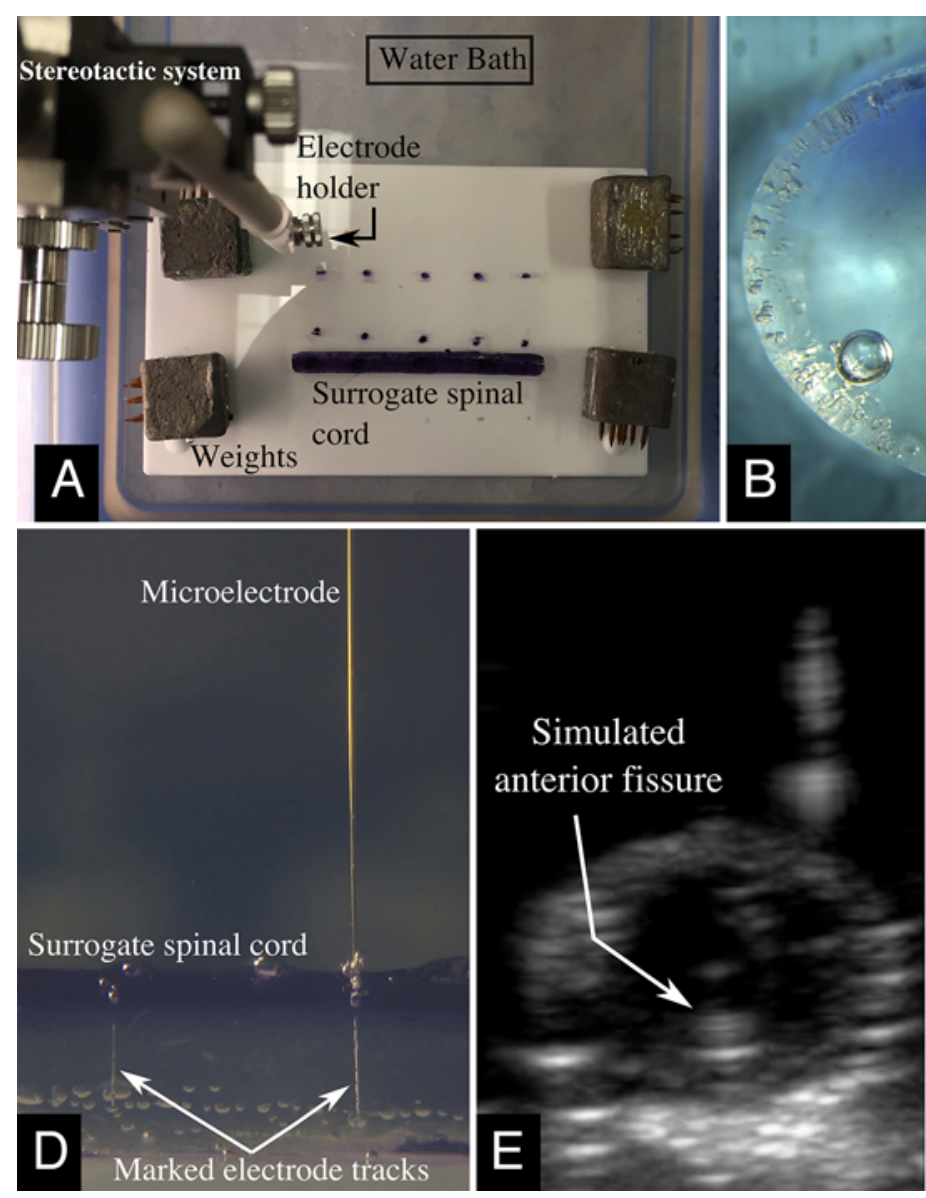

FIG. 7. Bench testing setup and methods. A: Setup used for bench tests: a surrogate spinal cord submerged in a saline bath with the stereotactic system positioned over it. Weights are used to prevent the plastic plate and the attached spinal cord from floating. B: Prior to testing, a partial cut was made on one side of the surrogate spinal cords to simulate the anterior fissure. The cut was made while the surrogate cords were in their molds to ensure the cuts were properly aligned with the major axis of the surrogate cords. This figure also shows, in a slice of gelatin spinal cord, an electrode track resulting from electrical stimulation through the implanted electrode. C: The electrode is implanted in the gelatin spinal cord. D: The electrode track and bubbles produced by stimulation through the electrode to mark the electrode's position for further analysis. E: Ultrasound image of the gelatin spinal cord and the electrode prior to its implantation in the transverse plane. F: Ultrasound image of the gelatin spinal cord and the electrode prior to its insertion in the sagittal plane. Figure is available in color online only.

\section{Benchtop Assessment of the Ultrasound-Guided Electrode Implantation Technique}

The ultrasound-guided electrode implantation technique was tested on the bench to assess its capabilities and limitations in a more controlled setting with a higher spatial resolution. The bench setup and the testing protocol not only directly measured the electrode insertion angle, but also allowed for reliable measurement of the targeting depth in the transparent surrogate cords (Fig. 7A). This is in contrast with the pig experiments in which the MR images were used to measure electrode insertion angles only.

Four gelatin hydrogel surrogate spinal cords were prepared as previously described, ${ }^{5}$ and a cut was made at their midline to simulate the anterior fissure (Fig. 7B). Hydrogel cords were selected because of their transparency and near physiological mechanical properties. The anterior side of the spinal cords was fixed onto a plastic plate (Fig. 7A), and the dorsal surface of the spinal cords was painted black to prevent the operator from using visual feedback for alignment. The plastic plate was then placed in a transparent container filled with saline for implantation.

A 125 - $\mu \mathrm{m}$-diameter platinum/iridium ( $\mathrm{Pt} / \mathrm{Ir}$ ) electrode (FHC Company) was used for insertion into the spinal cord. Alignment of the electrode prior to insertion was performed under ultrasound guidance (with a handheld probe) in both the transverse and sagittal planes (Fig. 7E and F). Landmarks used for identifying alignment of the surrogate spinal cords in the ultrasound images were the orientation of the dorsal surface and the orientation of the simulated anterior fissure. As in the pig experiments, the initial orientation of the electrode was randomly set before each trial.

After the electrode was aligned perpendicularly with respect to the spinal cord, the electrode was inserted to a depth of $4 \mathrm{~mm}$ using the micromanipulator. Electrical stimulation was used to mark the electrode track in the spinal cord for further analysis. Direct current (DC) stimulation at $20 \mathrm{~V}$ was delivered across the implanted 
electrode and a needle electrode placed in the saline bath. After stimulation was turned on, the electrode was slowly retracted from the spinal cord to mark the full length of the track (Fig. 7C and D).

A camera was positioned parallel to the sagittal plane of the cord to measure the insertion angle in this plane. In each surrogate spinal cord, three to five tracks were made from electrode insertions. After all insertions were completed and tracks were marked, the surrogate spinal cords were detached from their underlying plate and thin transverse sections were cut, each containing one electrode track. Microscopic images were taken from these sections to measure the insertion angle and the track length in the transverse plane (Fig. 7B). All angle measurements in the images were obtained using ImageJ software.

We conducted t-tests to compare alignment errors of the experiments conducted on the bench and those in the pigs in both the sagittal and transverse planes by using IBM SPSS software.

\section{Functional Testing of the Ultrasound-Guided Stereotactic System in a Live Pig}

In order to demonstrate the application of the ultrasound-guided spinal stereotactic system for intraoperative ISMS, an experiment was conducted in a domestic pig (weight $51 \mathrm{~kg}$ ). The surgical procedure was identical to the description provided in Surgery and Device Placement. A $100-\mu \mathrm{m} \mathrm{Pt} / \mathrm{Ir}$ electrode was used (FHC Co.), and the stimulation trains consisted of a frequency of $50 \mathrm{~Hz}$, pulse width of $200 \mu \mathrm{sec}$, and biphasic charge-balanced pulses with amplitudes of up to $150 \mu \mathrm{A}$. A needle return electrode was placed in the paraspinal muscles. In order to visualize movements generated by the ISMS, the animal's legs were suspended as shown in Fig. 8. For kinematic analysis of the generated movements, black marks were painted on the hip, knee, ankle, and metatarsophalangeal joints as well as on the iliac crest, and videos of the generated movements were recorded. Electromyographic (EMG) signals were also recorded using a Noraxon digital telemetry system and Ambu blue sensor electrodes (Noraxon Co.). Pairs of EMG electrodes were placed on the following muscles: rectus femoris, biceps femoris, lateral gastrocnemius, and tibialis anterior. The kinematics data were assessed using the MR3 (Noraxon Co.) and ImageJ software packages. The EMG data were analyzed using custom-written programs in Matlab (version 2015a, MathWorks Co.).

\section{Results}

\section{Findings Related to Ultrasound Imaging of the Spinal Cord}

An ultrasound probe tilted to an angle of $25^{\circ}-45^{\circ}$ in the transverse oblique scanning plane provided acceptable contrast for viewing the gray and white matter of the spinal cord. Careful cleaning of the surgical site was found to be important for achieving an acceptable image quality. Layers of blood clots forming on the dorsal surface of the cord or in the lateral recesses of the spinal canal are hyperechoic and can result in a distorted image.

The electrode and the glass markers were visualized in the ultrasound images of the spinal cord before and after implantation (Figs. 5A and 6B). There was no evident dif-

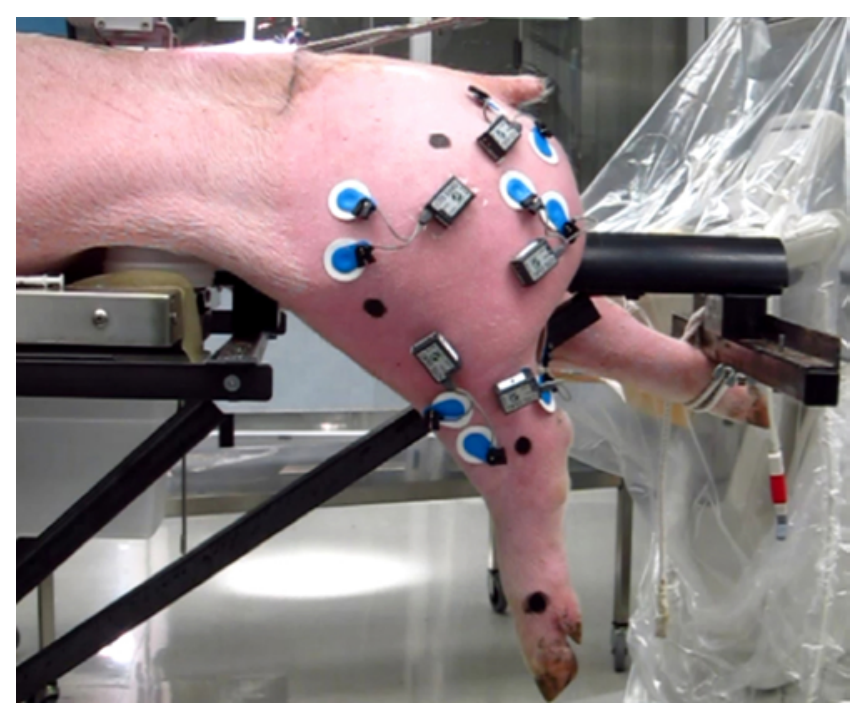

FIG. 8. Suspension setup used for the visualization of hind limb movements produced by the ISMS in the pig spinal cord. Figure is available in color online only.

ference in the quality of the ultrasound images of the gray and white matter between the experiments conducted in the cadavers and those conducted in the live animal. However, an additional way of visualizing the anterior fissure of the spinal cord was using Doppler imaging (Fig. 5C), which was only possible in the live animal.

\section{Assessment of Electrode Alignment in Pigs}

"Detection accuracy" of the electrode insertion angle was expressed as an error score defined by the difference between the marker insertion angle, measured from the MR image, and the needle insertion angle, measured from the ultrasound image. In calculating this error score, it was assumed that the marker insertion and careful tissue extraction (after the completion of the experiment) did not cause changes in the alignment of the marker. The detection accuracy was measured for 137 insertions in 6 animals in the transverse plane and for 36 insertions in 2 animals in the sagittal plane. The detection accuracy was $2.15^{\circ} \pm$ $0.38^{\circ}$ and $2.09^{\circ} \pm 0.35^{\circ}$ (mean $\pm 95 \%$ confidence interval) in the transverse and sagittal planes, respectively (Fig. 9).

"Alignment accuracy" of the electrode insertion was expressed as an error score defined by the difference between the measured insertion angle on the MR images and the target electrode orientation, which was perpendicular to the major axis of the spinal cord (Fig. 4). The spatial targeting error for a stereotactic system with degrees of freedom in the spherical coordinate system depends on the targeted depth. Therefore, to implement the $<0.5-\mathrm{mm}$ limit of the acceptable spatial targeting error for ISMS ${ }^{1,25}$ in the spherical coordinate system, the following equation was used:

$0.5>\sqrt{r^{\prime 2}+(r)^{2}-2(r) r^{\prime}\left[\sin \left(90^{\circ}\right) \sin \theta^{\prime} \cos \left(90^{\circ}-\varphi^{\prime}\right)+\cos \left(90^{\circ}\right) \cos \theta^{\prime}\right]}$

where $r$ is the depth of the target from the dorsal surface of the spinal cord, $r^{\prime}$ is the implanted length of the electrode, 


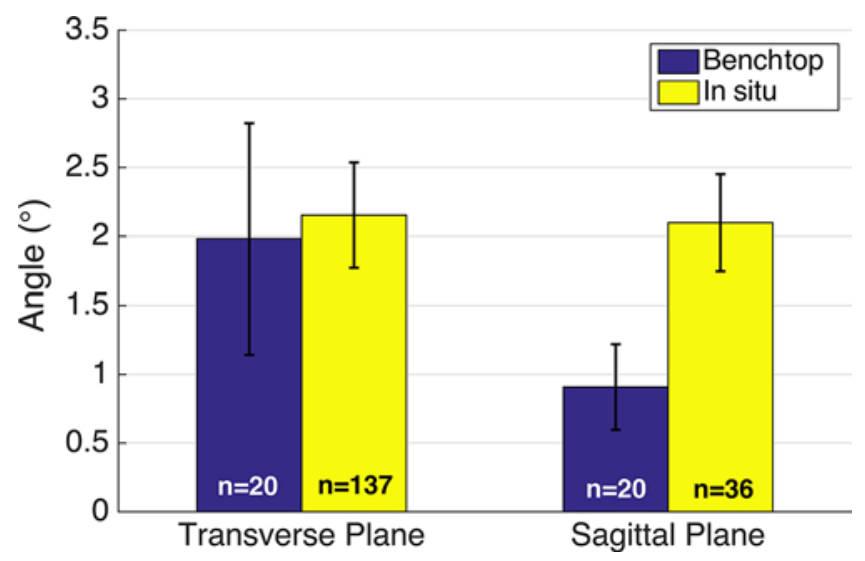

FIG. 9. Graph showing detection accuracy of the electrode insertion angle for intraoperative ultrasound imaging. Benchtop results reflect a comparison between the ultrasound measurements and the light microscopy measurements of the sample (Methods, Assessment of the Accuracy of Ultrasound-Guided Electrode Implantation in the Pig), and the in situ results reflect a comparison between the ultrasound and MRI measurements in the pigs (Methods, Surgery and Device Placement). Benchtop measurements were performed in 4 gelatin spinal cord samples, and measurements in the pigs were performed in 6 animals (transverse plane measurements performed in 6 animals and sagittal plane measurements performed in 2). Error bars represent the $95 \%$ confidence interval. Figure is available in color online only.

and $\varphi^{\prime}$ and $\theta^{\prime}$ are the electrode trajectory angles in the sagittal and transverse planes, respectively. The maximal depth of the ventral horns of the gray matter in the lumbar enlargement of the human spinal cord $(r)$ is approximately $6 \mathrm{~mm}^{35}$ from the dorsal surface.

In 6 pig experiments, a total of 225 markers were implanted in the spinal cords. Alignment in the sagittal plane was based on eyeballing and resulted in an overall alignment error of $1.4^{\circ} \pm 0.27^{\circ}$ (mean $\pm 95 \%$ confidence interval). Given the above equation, the approximately $1.5^{\circ}$ error measured for the alignment technique used in the sagittal plane results in a limit of approximately $4.5^{\circ}$ in the alignment error in the transverse plane for ISMS targets.

Electrode alignment in the transverse plane was conducted under two conditions (time constrained and unlimited time) using three different alignment techniques (eyeballing, ultrasound guidance using a handheld probe, and ultrasound guidance using a mounted probe). Alignment errors in the transverse plane are shown in Fig. 10. For both conditions, the alignment error using ultrasound guidance was significantly smaller than the one with the use of eyeballing ( $p<0.01$ for unlimited time, $p<0.001$ for time constrained). However, alignment errors were not significantly different between the two ultrasound guidance methods $(\mathrm{p}=0.99$ for unlimited time, $\mathrm{p}=0.84$ for time constrained). The upper bounds for the (95\%) confidence interval of the mean alignment error for ultrasound guidance with a handheld probe and ultrasound guidance with a mounted probe were $2.45^{\circ}$ and $2.63^{\circ}$, respectively, for the unlimited-time condition and $3.07^{\circ}$ and $3.57^{\circ}$, respectively, for the time-constrained condition. Overall, for targets $6 \mathrm{~mm}$ deep into the cord, the Cartesian equivalents of the maximal alignment errors under ultrasound guid- ance (time constrained, mounted probe for the transverse plane and eyeballing for the sagittal plane) were as follows: $0.37 \mathrm{~mm}$ in the mediolateral axis, $0.01 \mathrm{~mm}$ in the anteroposterior axis, and $0.17 \mathrm{~mm}$ in the craniocaudal axis.

In limiting the insertion time and the viewpoint for eyeballing and ultrasound guidance (only when the probe was fixed to the stereotactic setup), the alignment methods resulted in significantly larger alignment errors $(\mathrm{p}=$ 0.02 and 0.02 , respectively). No significant interaction was found between the condition (unlimited time, time constrained) and the alignment method $(\mathrm{p}=0.21)$.

\section{Benchtop Assessments}

Detection accuracy of the electrode insertion angle in the benchtop experiments was expressed by the difference between the measured insertion angle based on the ultrasound image and the measured angle from the microscopy image of the surrogate spinal cord section. The detection accuracies were $1.98^{\circ} \pm 0.84^{\circ}$ and $0.91^{\circ} \pm 0.31^{\circ}$ (mean \pm $95 \%$ confidence interval) in the transverse and sagittal planes, respectively (Fig. 9).

The bench setup not only allowed the measurement of alignment accuracy (deviation angles in transverse and sagittal planes as measured in pigs), but also allowed direct measurement of the spatial targeting error (3D distance from the target, which, in addition to the alignment error, also includes the error associated with implantation depth). Alignment accuracy of the inserted electrodes in the sagittal and transverse planes was $0.95^{\circ} \pm 0.50^{\circ}$ and $2.29^{\circ} \pm 0.81^{\circ}$ (mean $\pm 95 \%$ confidence interval), respectively (Fig. 11). Depth of the electrode tips in the transverse plane was $3.95 \pm 0.11 \mathrm{~mm}$. Based on these results, the spatial targeting error of ultrasound-guided implantation was calculated using the equation above (in this case, $r=4$ $\mathrm{mm}$ ) and was $0.22 \pm 0.02 \mathrm{~mm}$ (mean \pm standard deviation). In this study, these results were obtained for a surrogate cat spinal cord and a target depth of $4 \mathrm{~mm}$ for the electrode tip. Based on the measured deviation angles, if the targeting depth for lumbar enlargement of a human spinal cord were to be considered (maximal gray matter depth of $6 \mathrm{~mm})$, and assuming perfect depth targeting $\left(r=r^{\prime}=\right.$ $6 \mathrm{~mm}$ ), the calculated spatial targeting error (equation) remains below the $0.5-\mathrm{mm}$ limit. The upper boundary of the spatial targeting error in this case is calculated to be 0.36 $\mathrm{mm}$, using the upper boundaries of the $95 \%$ confidence intervals of the measured deviation angles for the electrode trajectory. The Cartesian equivalent of the maximal alignment errors in this case is as follows: $0.32 \mathrm{~mm}$ in the mediolateral axis, $0.01 \mathrm{~mm}$ in the anteroposterior axis, and $0.15 \mathrm{~mm}$ in the craniocaudal axis.

The alignment error in the benchtop experiments (ultrasound guidance) in the sagittal plane was significantly smaller than that in the experiments in pigs (assisted eyeballing method; $\mathrm{p}=0.01$ ). However, alignment errors in the transverse plane (using ultrasound guidance) were not significantly different from each other $(\mathrm{p}=0.83)$.

\section{Intraoperative ISMS Using the Spinal Stereotactic System}

Lumbar enlargement of the spinal cord of a live pig was intraoperatively mapped using the ultrasound-guided spinal stereotactic system. In this experiment, 21 locations 


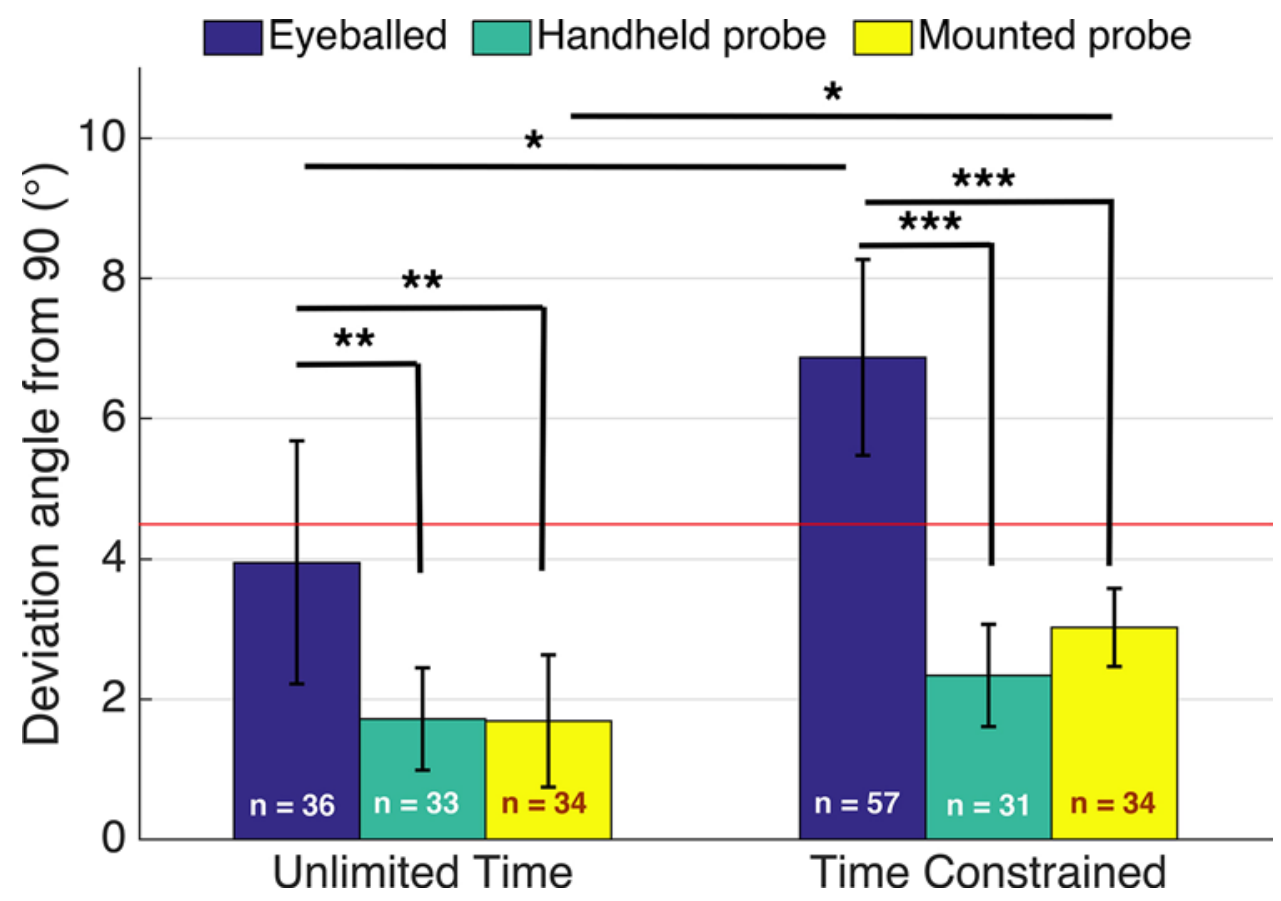

FIG. 10. Alignment accuracy of the implant in the transverse plane of the spinal cord, as measured in pigs. Implantations conducted under the unlimited-time condition were not limited in time and the surgeon was allowed to move around the subject freely to obtain different viewpoints of the implant site. Conversely, the time-constrained condition simulated a more realistic surgical scenario in which the implantation time for each electrode was limited to 90 seconds and the surgeon was only allowed to move on one side of the subject. The red horizontal line represents the limit up to which a deviation angle from $90^{\circ}$ is acceptable for ISMS applications in the human spinal cord. Error bars represent the $95 \%$ confidence interval. ${ }^{*} p \leq 0.05 ;{ }^{* *} p \leq 0.01 ;{ }^{* * *} p \leq 0.001$. Figure is available in color online only.

were electrically stimulated in the spinal cord, resulting in functional movements in the leg. The generated movements included both single joint movements, such as knee extension and ankle extension, and multi-joint synergistic movements, such as extensor synergy involving the hip, knee, and ankle. An example of the movements and EMG signals produced by ISMS in one location is shown in Fig. 12. The observed functional organization of the mapped region of the spinal cord in this animal was consistent with functional maps for the lumbar spinal cords of pigs, ${ }^{10}$ cats, ${ }^{26,27}$ and rhesus monkeys. ${ }^{39}$

\section{Discussion}

In this study, we proposed and evaluated a new spinal stereotactic system that relies on real-time (i.e., instantaneous) ultrasound guidance for accurate targeting inside the gray matter of the spinal cord. Comparisons conducted with traditional visual targeting methods demonstrated the superior performance of ultrasound-guided targeting.

Emerging intraspinal interventions for spinal cord repair and restoration of lost functions require appropriate neurosurgical setups and tools that ensure their safe and successful clinical implementation. Despite advances in this area, ${ }^{3,8,19,20}$ important challenges remain in reaching submillimeter-sized targets within the spinal cord. The ability of intraoperative ultrasound imaging to visualize the gray and white matter of the spinal cord can not only guide electrode alignment and target selection prior to im- plantation, but also provide feedback during and after insertion. This is not possible with existing MRI-guided stereotactic systems because of the inherent restrictions and challenges of MRI compared to those of ultrasound imaging. Examples of such challenges include imaging artifacts resulting from metallic instrumentation and implants, as

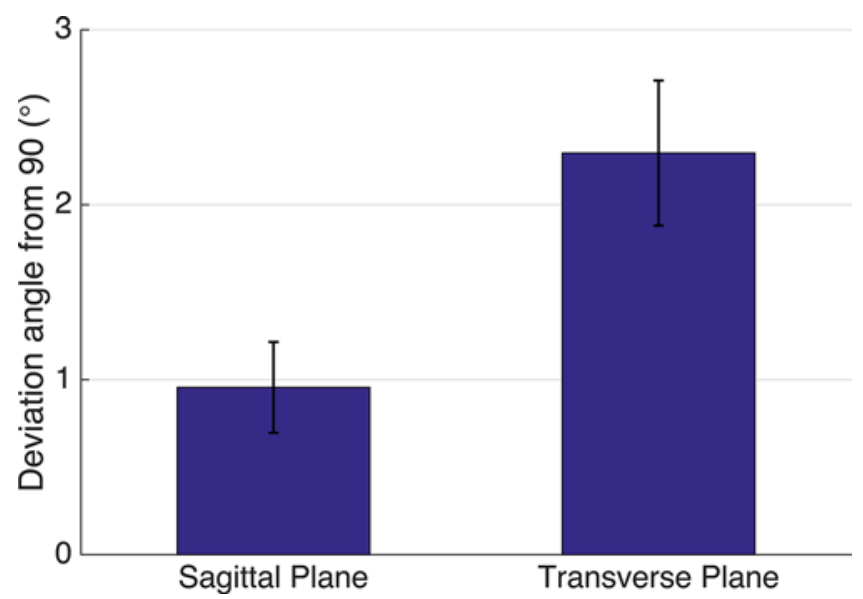

FIG. 11. Alignment accuracy of the implant in the spinal cord, as measured on the bench. Electrodes were placed using ultrasound guidance (handheld probe) in both planes and consistent with the timeconstrained condition (number of implanted markers under this condition $=17$ ). Figure is available in color online only. 

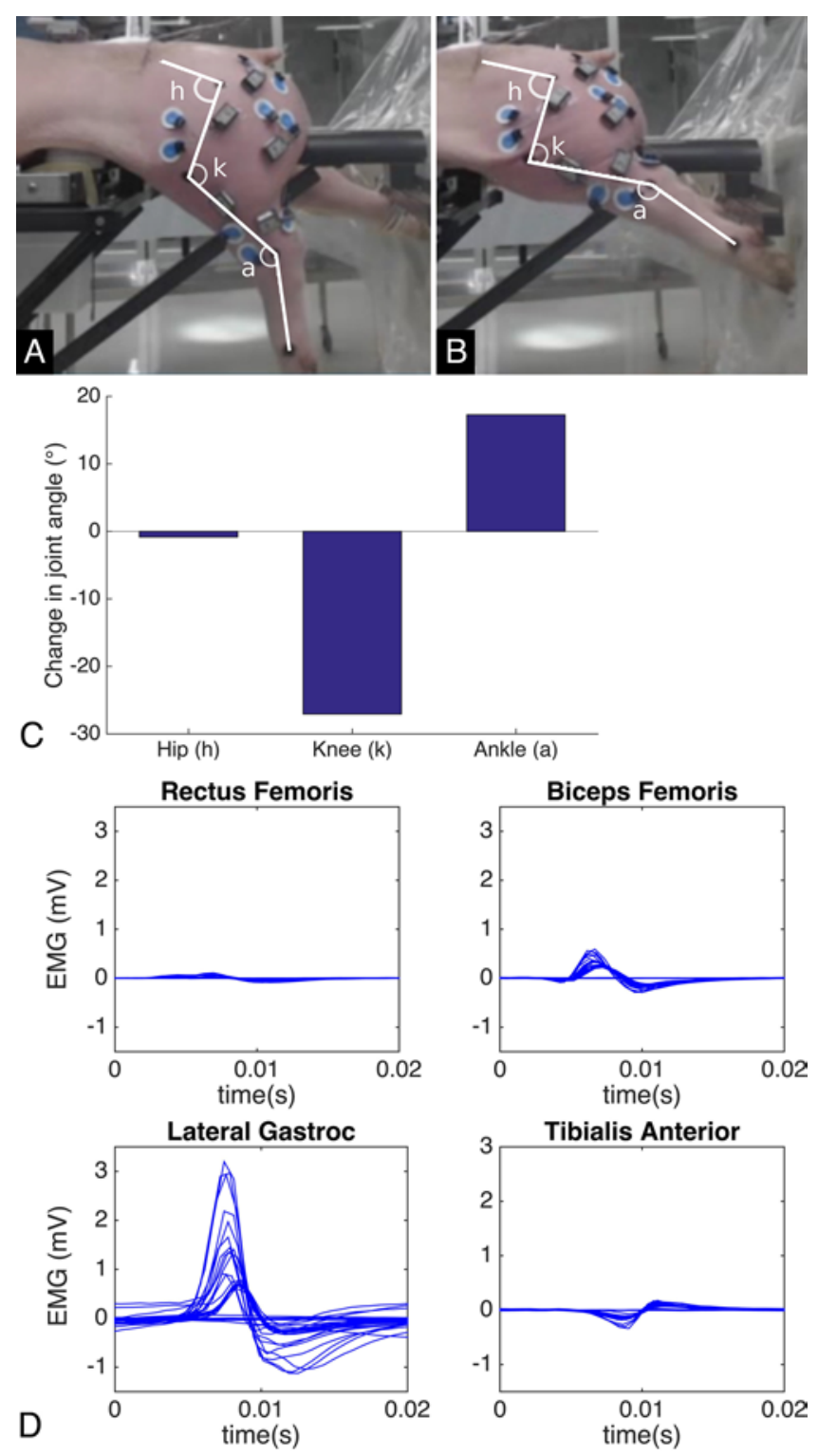

FIG. 12. Suspension setup used for intraoperative testing of ISMS in the pig spinal cord and an example of the results produced. A: Leg position before stimulation. B: Leg position after stimulation at an amplitude of 75 $\mu \mathrm{A}$. C: Joint angle changes as a result of the generated movement. D: Evoked EMG activity. Gastroc = gastrocnemius muscle. Figure is available in color online only.

well as the safety concerns associated with possible current induction through them. The latter is critical in ISMS since the procedure utilizes an electrically active implant consisting of an array of microelectrodes, lead wires, connectors, and a wireless stimulator. In the context of ISMS, another limitation of currently available MRI-guided systems is the fact that the size of the laminectomy region is driven by the size of the MRI coils. This is because the MRI coil is placed in the exposed region over the spinal cord for the best image quality. For example, the system developed by the Mayo Clinic ${ }^{9}$ utilizes a custom-built coil that is $7.5 \mathrm{~cm}$ long; therefore, the length of the laminectomy must be larger than that. This contrasts with the in- traoperative ultrasound imaging probe used in the present study, which had a footprint of $2.3 \mathrm{~cm}$. As image fusion software platforms become more available for ultrasound and MRI, ${ }^{15,17,18}$ ultrasound imaging of the spinal cord in conjunction with preoperative MRI has the potential to provide even more information intraoperatively.

Detection error of the insertion angle using ultrasound imaging was, on average, less than $2.2^{\circ}$ in the transverse plane, both in pigs and on benchtop testing. However, as shown in Fig. 9, the detection error in the sagittal plane with respect to MRI in pigs was higher than that of the direct benchtop measurements. This difference may be partly attributable to the lower MRI resolution obtained in the sagittal plane $(0.31 \times 0.31 \mathrm{~mm}$ in the sagittal plane vs $0.25 \times 0.25 \mathrm{~mm}$ in the transverse plane). The importance of detection accuracy is in its relationship to targeting accuracy. The targeting error (independent of any set target angle) is the sum of the detection error of the insertion angle, the errors involved in performing the alignment using the stereotactic hardware, and the errors involved in the implantation process. Therefore, the value of detection accuracy provides a best-case scenario for the targeting accuracy of the ultrasound-guided alignment.

Targeting resolution of ultrasound-guided electrode insertion was also assessed both in pigs and on the benchtop. In the pig experiments, ultrasound guidance was only used in the transverse plane because of the spatial limitations imposed by the glass-marker insertion tool. Nevertheless, ultrasound guidance (transverse plane) in conjunction with eyeballing (sagittal plane) was able to meet the targeting error limit of $<0.5 \mathrm{~mm}$ for ISMS (Fig. 10). A comparison between the alignment methods used in the transverse plane demonstrated the superior targeting accuracy of ultrasound guidance compared with eyeballing. The targeting accuracy through eyeballing did not meet the targeting goal for ISMS.

The two ultrasound-guided alignment methods provided similar targeting accuracies. However, when the ultrasound probe was fixed to the stereotactic setup, the surgeon could use both hands to make the required adjustments to the micromanipulator stages for a desired alignment. Limiting the electrode alignment time and the surgeon's viewpoint resulted in larger targeting errors for both the eyeballing and ultrasound-guided alignment methods. However, the ultrasound-guided alignment methods under these conditions still met the targeting accuracy goal set for ISMS.

Comparison between the targeting accuracy results of eyeballing in the transverse and sagittal planes shows a larger error in the transverse plane than in the sagittal plane. This finding may be explained by the transverse curvature of the spinal cord, which makes visual alignment more difficult. It is also worth noting that the spatial limitation that prevented the use of ultrasound imaging in pigs in the sagittal plane (design of the glass-tube insertion tool) does not exist for ISMS in which the glass-tube insertion tool is replaced with the electrode stylus (Fig. 2A). The glass-tube insertion tool was used only for the purpose of placing markers in the spinal cord to allow subsequent analysis with MRI.

Benchtop experiments utilized the electrode stylus and 
ultrasound guidance for alignment of the electrode in both the transverse and sagittal planes. The spatial targeting error for these experiments was $<0.23 \mathrm{~mm}$ (upper bound of its $95 \%$ confidence interval) for a target $4 \mathrm{~mm}$ below the surface. This targeting resolution is approximately 5 times higher than the values reported for an existing MRI-guided system $(1.09 \pm 0.2 \mathrm{~mm}){ }^{9}$ Ultrasound-guided alignment in the transverse plane in the benchtop experiments was performed similarly to the experiments in pigs. Ultrasound guidance in the sagittal plane in the benchtop experiments provided higher alignment accuracy than eyeballing in the pigs.

Although this study focused only on the application of intraoperative ISMS, the proposed setup has the potential to be used for implantation of ISMS arrays for chronic use as well. This may be realized by using a modified electrode-holder design that can release the electrodes after implantation. The bench setup used in this study can be utilized as a training tool for users of the proposed image-guided stereotactic system. It can also provide a highthroughput testing apparatus of the targeting accuracy of various intraspinal interventions.

The proposed spinal stereotactic system is easy to assemble and remove and is compatible with existing surgical instrumentation. The system can be sterilized using gas sterilization methods such as vaporized hydrogen peroxide (VHP) and ethylene oxide (EtO) sterilization or other low-temperature sterilization techniques, such as STERRAD (Johnson \& Johnson, data not shown). Also, since the stereotactic setup is fixed onto the spine through pedicle screws, its application is not limited to a specific region of the spine. Additionally, the design of the stereotactic setup provides spinal fixation at the implantation site. This is especially beneficial for applications such as intraoperative ISMS in which movements produced in the leg can result in unwanted movements in the spine and spinal cord.

Nonetheless, since this system mounts onto pedicle screws, a limitation of its application may be in people with compromised bone strength. In such cases, the addition of infra-laminar hooks on the levels above and below the laminectomy region may be considered. ${ }^{6,22}$ Also, in such cases, the proposed stereotactic system allows for the addition of a counterweight over the loops to balance the weight of the micromanipulator system and minimize the torque transferred to the spine.

\section{Conclusions}

In this study, a novel ultrasound-guided spinal stereotactic system for ISMS was developed. The system is mounted onto the spine and has 6 degrees of freedom with a sufficient range of motion that allows precise targeting of various parts of the spinal cord. Guidance provided by intraoperative ultrasound imaging can assist with localizing the implant site and accurately guiding the electrode trajectory.

This system can be used to perform procedures on the spinal cord that require high precision and accurate location and do not tolerate relative movement between the device and the spinal cord. Examples of such procedures are injections (e.g., stem cells, drugs) into the spinal cord or insertion of electrodes into the spinal cord for electrical stimulation. This system will be used in the first clinical tests of ISMS, thus facilitating the clinical translation of this electrical stimulation modality for restoring standing and walking after spinal cord injury.

\section{Acknowledgments}

We thank Mr. Rod Gramlich for modification of the surgical table for pigs to allow for the suspension of the animal's hind limbs. We also thank Ms. Bethany Kondiles for her contribution to the design of the marker insertion tool and her assistance with the pilot experiments and Ms. Ashley Dalrymple and Dr. Leandro Solis for their assistance with the data collection process during pilot experiments.

Funding for this project was provided by the Canadian Institutes of Health Research (Open Operating Grants Program; MOP \#130520) and the US Army (Congressionally Directed Medical Research Program-Spinal Cord Injury Research Program; \#SC110029); A.T. was supported by a Vanier Canada Graduate Scholarship and an Alberta Innovates-Health Solutions Graduate Studentship; and V.K.M. was an Alberta Heritage Foundation for Medical Research Senior Scholar.

\section{References}

1. Bagshaw EV, Evans MH: Measurement of current spread from microelectrodes when stimulating within the nervous system. Exp Brain Res 25:391-400, 1976

2. Bamford JA, Mushahwar VK: Intraspinal microstimulation for the recovery of function following spinal cord injury. Prog Brain Res 194:227-239, 2011

3. Blanquer M, Pérez-Espejo MA, Martínez-Lage JF, Iniesta F, Martinez S, Moraleda JM: A surgical technique of spinal cord cell transplantation in amyotrophic lateral sclerosis. J Neurosci Methods 191:255-257, 2010

4. Busscher I, Ploegmakers JJW, Verkerke GJ, Veldhuizen AG: Comparative anatomical dimensions of the complete human and porcine spine. Eur Spine J 19:1104-1114, 2010

5. Cheng C, Kmech J, Mushahwar VK, Elias AL: Development of surrogate spinal cords for the evaluation of electrode arrays used in intraspinal implants. IEEE Trans Biomed Eng 60:1667-1676, 2013

6. DeWald CJ, Stanley T: Instrumentation-related complications of multilevel fusions for adult spinal deformity patients over age 65: surgical considerations and treatment options in patients with poor bone quality. Spine (Phila Pa 1976) 31 (19 Suppl):S144-S151, 2006

7. Federici T, Hurtig CV, Burks KL, Riley JP, Krishna V, Miller BA, et al: Surgical technique for spinal cord delivery of therapies: demonstration of procedure in Gottingen minipigs. J Vis Exp (70):4371, 2012

8. Féron F, Perry C, Cochrane J, Licina P, Nowitzke A, Urquhart S, et al: Autologous olfactory ensheathing cell transplantation in human spinal cord injury. Brain 128:2951-2960, 2005

9. Grahn PJ, Goerss SJ, Lujan JL, Mallory GW, Kall BA, Mendez AA, et al: MRI-guided stereotactic system for delivery of intraspinal microstimulation. Spine (Phila Pa 1976) 41:E806-E813, 2016

10. Hachmann JT, Jeong JH, Grahn PJ, Mallory GW, Evertz LQ, Bieber AJ, et al: Large animal model for development of functional restoration paradigms using epidural and intraspinal stimulation. PLoS One 8:e81443, 2013

11. Holinski BJ, Mazurek KA, Everaert DG, Toossi A, LucasOsma AM, Troyk P, et al: Intraspinal microstimulation produces over-ground walking in anesthetized cats. J Neural Eng 13:056016, 2016

12. Ihnatsenka B, Boezaart AP: Ultrasound: basic understanding 
and learning the language. Int J Shoulder Surg 4:55-62, 2010

13. Lamanna JJ, Urquia LN, Hurtig CV, Gutierrez J, Anderson $C$, Piferi P, et al: Magnetic resonance imaging-guided transplantation of neural stem cells into the porcine spinal cord. Stereotact Funct Neurosurg 95:60-68, 2017

14. Lau B, Guevremont L, Mushahwar VK: Strategies for generating prolonged functional standing using intramuscular stimulation or intraspinal microstimulation. IEEE Trans Neural Syst Rehabil Eng 15:273-285, 2007

15. Lee MW: Fusion imaging of real-time ultrasonography with CT or MRI for hepatic intervention. Ultrasonography 33:227-239, 2014

16. Lima C, Escada P, Pratas-Vital J, Branco C, Arcangeli CA, Lazzeri G, et al: Olfactory mucosal autografts and rehabilitation for chronic traumatic spinal cord injury. Neurorehabil Neural Repair 24:10-22, 2010

17. Logan JK, Rais-Bahrami S, Turkbey B, Gomella A, Amalou $\mathrm{H}$, Choyke PL, et al: Current status of magnetic resonance imaging (MRI) and ultrasonography fusion software platforms for guidance of prostate biopsies. BJU Int 114:641652,2014

18. Marks L, Young S, Natarajan S: MRI-ultrasound fusion for guidance of targeted prostate biopsy. Curr Opin Urol 23:43-50, 2013

19. Mazzini L, Ferrero I, Luparello V, Rustichelli D, Gunetti M, Mareschi K, et al: Mesenchymal stem cell transplantation in amyotrophic lateral sclerosis: a Phase I clinical trial. Exp Neurol 223:229-237, 2010

20. Medani K, Riley J, Lamanna J, Boulis N: Spinal cord cellular therapeutics delivery: device design considerations, in Childers MK (ed): Regenerative Medicine for Degenerative Muscle Diseases, Stem Cell Biology and Regenerative Medicine. New York: Springer, 2016, pp 109-127

21. Mercier LM, Gonzalez-Rothi EJ, Streeter KA, Posgai SS, Poirier AS, Fuller DD, et al: Intraspinal microstimulation and diaphragm activation after cervical spinal cord injury. J Neurophysiol 117:767-776, 2017

22. Murakami H, Tsai KJ, Attallah-Wasif ES, Yamazaki K, Shimamura T, Hutton WC: A biomechanical assessment of infra-laminar hooks as an alternative to supra-laminar hooks in thoracolumbar fixation. Spine (Phila Pa 1976) 31:967-971, 2006

23. Mushahwar VK, Collins DF, Prochazka A: Spinal cord microstimulation generates functional limb movements in chronically implanted cats. Exp Neurol 163:422-429, 2000

24. Mushahwar VK, Horch KW: Muscle recruitment through electrical stimulation of the lumbo-sacral spinal cord. IEEE Trans Rehabil Eng 8:22-29, 2000

25. Mushahwar VK, Horch KW: Proposed specifications for a lumbar spinal cord electrode array for control of lower extremities in paraplegia. IEEE Trans Rehabil Eng 5:237243, 1997

26. Mushahwar VK, Horch KW: Selective activation and graded recruitment of functional muscle groups through spinal cord stimulation. Ann N Y Acad Sci 860:531-535, 1998

27. Mushahwar VK, Horch KW: Selective activation of muscle groups in the feline hindlimb through electrical microstimulation of the ventral lumbo-sacral spinal cord. IEEE Trans Rehabil Eng 8:11-21, 2000

28. Nashold BS, Friedman H, Grimes J: Electrical stimulation of the conus medullaris to control bladder emptying in paraplegia: a ten-year review. Appl Neurophysiol 45:40-43, 1982

29. Peckham PH, Knutson JS: Functional electrical stimulation for neuromuscular applications. Annu Rev Biomed Eng 7:327-360, 2005

30. Riley J, Federici T, Polak M, Kelly C, Glass J, Raore B, et al:
Intraspinal stem cell transplantation in amyotrophic lateral sclerosis: a phase I safety trial, technical note, and lumbar safety outcomes. Neurosurgery 71:405-416, 2012

31. Riley J, Glass J, Feldman EL, Polak M, Bordeau J, Federici $\mathrm{T}$, et al: Intraspinal stem cell transplantation in amyotrophic lateral sclerosis: a phase I trial, cervical microinjection, and final surgical safety outcomes. Neurosurgery 74:77-87, 2014

32. Riley JP, Raore B, Taub JS, Federici T, Boulis NM: Platform and cannula design improvements for spinal cord therapeutics delivery. Neurosurgery 69:ons147-ons155, 2011

33. Saberi H, Moshayedi P, Aghayan HR, Arjmand B, Hosseini SK, Emami-Razavi SH, et al: Treatment of chronic thoracic spinal cord injury patients with autologous Schwann cell transplantation: an interim report on safety considerations and possible outcomes. Neurosci Lett 443:46-50, 2008

34. Saigal R, Renzi C, Mushahwar VK: Intraspinal microstimulation generates functional movements after spinal-cord injury. IEEE Trans Neural Syst Rehabil Eng 12:430-440, 2004

35. Sengul G, Watson C, Tanaka I, Paxinos G: Atlas of the Spinal Cord: Mouse, Rat, Rhesus, Marmoset, and Human, ed 1. Cambridge, MA: Academic Press, 2012

36. Sheng SR, Wang XY, Xu HZ, Zhu GQ, Zhou YF: Anatomy of large animal spines and its comparison to the human spine: a systematic review. Eur Spine J 19:46-56, 2010

37. Sunshine MD, Cho FS, Lockwood DR, Fechko AS, Kasten MR, Moritz CT: Cervical intraspinal microstimulation evokes robust forelimb movements before and after injury. $\mathbf{J}$ Neural Eng 10:036001, 2013

38. Toossi A, Everaert DG, Azar A, Dennison CR, Mushahwar VK: Mechanically stable intraspinal microstimulation implants for human translation. Ann Biomed Eng 45:681-694, 2017

39. Toossi A, Everaert DG, Perlmutter SI, Mushahwar VK: Functional organization of motoneuronal pools in the lumbar spinal cord of monkeys: intraspinal microstimulation targets, in Where Science Meets Innovation: A Joint Meeting of NANS \& NIC. Chicago: North American Neuromodulation Society, 2016 (Abstract) (https://www.neuromodulation.org/ Portals/0/NANS16_NIC_Syllabus_final.pdf) [Accessed February 28,2018$]$

40. Zimmermann JB, Jackson A: Closed-loop control of spinal cord stimulation to restore hand function after paralysis. Front Neurosci 8:87, 2014

\section{Disclosures}

Medtronic Inc. provided the pedicle screws at no charge. FHC Inc. provided microelectrodes at no cost. Philips Inc. loaned the ultrasound probe to the project at no cost.

\section{Author Contributions}

Conception and design: Mushahwar, Toossi, Everaert, Jaremko, Kao, Konrad. Acquisition of data: Mushahwar, Toossi, Everaert, Seres, Jaremko, Robinson. Analysis and interpretation of data: Mushahwar, Toossi, Robinson. Drafting the article: Toossi. Critically revising the article: Mushahwar, Toossi, Everaert, Jaremko, Konrad. Reviewed submitted version of manuscript: all authors. Approved the final version of the manuscript on behalf of all authors: Mushahwar. Statistical analysis: Toossi. Study supervision: Mushahwar.

\section{Correspondence}

Vivian K. Mushahwar: University of Alberta, Edmonton, AB, Canada.vivian.mushahwar@ualberta.ca. 